\title{
Faits et logique
}

\author{
Fernand Labrie
}

$\mathbf{N}$ otre article publié dans The Joumal of Urology est la première et la seule étude à ce jour sur l'incidence du cancer de la prostate dans une population d'hommes choisis au hasard et sans symptomatologie prostatique particulière. Elle est également la première et la seule étude sur l'évaluation de l'efficacité relative des trois techniques actuellement disponibles pour le diagnostic du cancer de la prostate au stade précoce, soit la mesure du PSA sérique, le toucher rectal et l'échographie transrectale de la prostate dans une telle population. Les conclusions de cette étude ne laissent pas de place à l'hypothèse. Par ailleurs, tout autre discours ne peut être plus qu'une supposition dont il s'agit de mesurer le bien-fondé.

Dans l'interprétation et dans l'utilisation des données de notre étude, l'on doit tenir rigoureusement compte des connaissances les plus significatives obtenues au cours des demières années dans le domaine du cancer de la prostate. Parmi les observations les plus conséquentes, l'on doit reconnaître les résultats des remarquables études de T.A. Stamey et al. [1] qui ont observé que les tumeurs prostatiques de petit volume $(<3 \mathrm{~g}$ ou diamètre moyen de $15 \mathrm{~mm}$ ) ont peu de chances de métastaser, sont mieux différenciées, ont peu de chances d'envahir la capsule et sont donc guérissables par prostatectomie radicale [2]. Ces données fort importantes viennent à l'appui de la suggestion que la seule chance d'offrir la guérison au patient souffrant du cancer de la prostate est de pouvoir traiter ce cancer pendant qu'il est encore localisé au niveau de la prostate. Cette fenêtre mentionnée dans mon article réfère donc à ces données maintenant bien reconnues [2]. Paul Robel et Alain Jardin s'inquiètent de "l'impos- cis le temps écoulé entre l'apparition de la première cellule néoplasique et la possibilité de détection du cancer de la prostate ". Ces auteurs doivent reconnaître que l'on doit prendre des décisions fondées sur l'ensemble d'une population tout en sachant très bien qu'il existe des variations entre individus. Il est donc logique de croire que la chance de guérison réside dans le diagnostic et le traitement de "petits" cancers, peu importe la durée exacte du développement de ce cancer. D'ailleurs, personne ne pourra jamais répondre à cette question.

Concernant le coût du dépistage avec le test de PSA, il est estimé à $1500 \$$ pour le premier dépistage. Bien entendu, l'incidence du cancer va diminuer lors des dépistages ultérieurs. Toutefois, une augmentation de $100 \%$ ou même de $200 \%$ du coût demeure encore bien en deçà des coûts déjà acceptés pour le dépistage du cancer du sein qui sont estimés à plus de $30000 \$$ par nouveau cas de cancer. Le décès par cancer de la prostate serait-il si peu important? Quant aux cancers impossibles à dépister, je ne vois pas de relation avec le coût !

Insistons maintenant sur certains faits bien connus en urologie qui devraient satisfaire les points mentionnés par Paul Robel et Alain Jardin.

\section{Age du dépistage}

Suite à nos données, notre recommandation est le dépistage à partir de l'âge de 50 ans et non de 45 ans. D'ailleurs, la même limite d'âge et la même fréquence de dépistage est recommandée par l'American Urological Association et I'American College of Radiology. J'ai trop vu de cancers métastasiques chez des hommes dans la cinquantaine pour accepter la suggestion de P. Robert et A. Jardin d'attendre 60 ans avant de penser au dépistage.

\section{Nombre de biopsies}

Dans le cas d'un niveau de PSA sérique au-dessus de $3 \mu \mathrm{g} / \mathrm{l}$, l'approche maintenant utilisée est d'évaluer d'abord le volume de la prostate afin de vérifier si l'augmentation du niveau de PSA peut être expliquée par l'hyperplasie bénigne de la prostate, ce qui élimine un bon nombre de biopsies. A notre clinique du cancer de la prostate, le taux de biopsies positives est de $40 \%$ contrairement au faible taux de 1 sur $6(17 \%)$ indiqués par P. Robel et A. Jardin. Le récent article de F. Lee et al. [2] décrit un taux de biopsies positives de $46 \%$. Ce taux me semble fort acceptable. Nous ne recommandons la biopsie que lorsque l'échographie est suggestive de cancer ou que le PSA élevé est inexpliqué par le volume de la prostate ou que le toucher rectal est positif, ce qui limite grandement le nombre de biopsies négatives.

3. Bénéfices du diagnostic d'un cancer de la prostate limité à la glande Il est étonnant de lire l'interprétation faite par P. Robel et A. Jardin de l'article de Johansson publié dans le JAMA d'avril 1992 [3]. Je suis surpris qu'ils n'aient pas analysé de façon plus critique les données de Johansson qui offrent un bel exemple d'un article où les conclusions ne correspondent pas aux données. Il est dangereux de lire seulement le résumé d'un article. Dans ce cas-ci, les faits sont bien différents et ils sont les suivants : 223 patients porteurs de cancer localisé $\mathrm{T}_{0,1,2}$ ont été sujets à un suivi potentiel de 10 ans. Parmi ceux qui ne sont pas décédés (l'âge moyen au départ était de 72 ans), 74 (34\%) ont montré une progression de la maladie au moins à travers la capsule prostatique et 26 d'entre eux $(35 \%)$ ont développé des métastases. Le titre de cet article est d'ailleurs inexact car $84 \%$ des patients 
ont été traités, ce qui explique probablement en grande partie les "bons" résultats obtenus. De fait, contrairement à l'interprétation de $\mathrm{P}$. Robel et A. Jardin, cet article offre un exemple plutôt convaincant de l'avantage de traiter le cancer de la prostate au stade précoce (voir figure 4 de Johansson). En effet, seulement $15,8 \%$ des patients traités au stade précoce ont montré une progression sur une période de cinq ans comparativement à 77,2 \% pour ceux ayant déjà des métastases au moment du traitement hormonal. A noter que le risque de progression était 10,3 fois plus élevé si le traitement hormonal était effectué au stade de métastases. Dix-neuf $(8,5 \%)$ patients sont morts de cancer. Compte tenu que près de la moitié (109) sont morts d'autres causes en raison de l'âge avancé au moment d'entrer dans l'étude, il y a lieu de multiplier le risque de décès dus au cancer de la prostate, soit $8,5 \%$, par un facteur d'au moins 2, si l'on veut appliquer cette étude à des hommes plus jeunes, soit entre 50 et 70 ans. Comme ces patients ont été suivis régulièrement, en majorité traités avant l'apparition des métastases, le taux de décès présenté est grandement sous-estimé. En d'autres termes, les hommes étaient trop âgés pour vivre assez longtemps, dans environ la moitié des cas, et pour mourir du cancer de la prostate. Il s'agit ici du groupe d'âge où la décision de traitement est la plus difficile mais les $12 \%$ de chances de développer des métastases et les $8,5 \%$ de chances de mourir du cancer de la prostate pour un homme de 72 ans ne sont pas un faible risque et la décision de traitement, surtout antihormonal, va dépendre grandement de l'état général et de l'espérance de vie de chaque patient. Le chiffre le plus significatif est que $34 \%$ ont montré un cancer qui a progressé à l'extérieur de la capsule prostatique pendant la période de 10 ans de suivi et qu'une correction pour le grand nombre de décès dus à l'âge avancé nous indique que près de $60 \%$ auraient probablement montré une progression pendant les 10 premières années de suivi si l'on avait étudié un groupe d'hommes âgés de 50 à 70 ans au lieu de patients âgés en moyenne de 72 ans au départ de l'étude.

Il est également bon de considérer les résultats de A. Larson et B.J. Noren [4]. Des 31 patients avec tumeurs grade $T_{1,2}, 24(77 \%)$ ont montré une progression de la maladie avant cinq ans, dont 16 (52\%) avec métastases. Dans le même ordre d'idée, il faut mentionner les données d'Epstein et al. [5], obtenues chez 94 hommes avec cancer de la prostate à un stade encore plus précoce, soit au stade $\mathrm{A}_{1+}$. A huit ans, alors que seulement $50 \%$ étaient encore vivants, le cancer avait progressé dans huit cas (16\%) et six $(12 \%)$ étaient décédés du cancer de la prostate.

Comme le cancer de la prostate est la deuxième cause de décès par cancer en Amérique du Nord et dans la plupart des pays d'Europe, l'on devrait se rendre à l'évidence que ce cancer n'est pas bénin et que l'approche actuellement utilisée n'est pas satisfaisante. Notre article décrit une stratégie de dépistage du cancer efficace, objective, peu coûteuse, en général, et facilement acceptable pour la population [6]. Il ne faut pas non plus oublier que les cancers de la prostate découverts avec les techniques diagnostiques actuelles ne sont pas vraiment de petits cancers car leur diamètre est de plus de $5 \mathrm{~mm}$ et, généralement, de l'ordre de 1 à $1,5 \mathrm{~cm}$ de diamètre. Il semble logique de croire les données de Jewett [7] et de beaucoup d'autres groupes [8] qui ont conclu que "les patients traités par prostatectomie radicale pour des cancers limités à la prostate ont une survie comparable à celle des hommes du même âge ne souffrant pas du cancer de la prostate ". Même si les données sur le cancer de la prostate au stade précoce sont limitées, il semble difficile de ne pas suivre cette logique. Agir autrement permettra au cancer de la prostate de demeurer encore pendant de nombreuses années le deuxième cancer le plus meurtrier chez l'homme. Comme l'admettent en conclusion P. Robert et A. Jardin, le tirage au sort entre l'absence de traitement et la prostatectomie radicale est difficile à proposer au plan de l'éthique. Alors, pourquoi ne pas entreprendre sur une plus grande échelle des études prospectives et randomisées afin d'évaluer les traitements anti-hormonal néoadjuvant et adjuvant associés à la prostatectomie radicale [9] telles que celles effectuées à notre clinique de la prostate au Cen- tre hospitalier de l'université Laval à Québec, de même qu'aux États-Unis et en Europe. Il est urgent qu'un plus grand nombre de centres urologiques se joignent à ces groupes et orientent leurs recherches vers le domaine probablement le plus prometteur qui est celui du traitement du cancer de la prostate au stade précoce. Avant tout ceci, il faut cependant pouvoir diagnostiquer le cancer de la prostate au stade précoce, car autrement, l'on continuera à le traiter au stade avancé et le cancer de la prostate réussira peut-être à devancer le cancer du poumon comme cause de décès dus au cancer

\section{Fernand Labrie}

Directeur scientifique, Centre hospilalier de l'université Laval, 2705 boulevard Lauria Queboc, 61 V4G2 Canada

\section{RÉFÉRENCES}

1. McNeal JE, Villicr AA, Redwine FA, Freiha FS, Stamey TA. Histological differentiation, cancer volume and pelvic lymph node metastasis in adenocarcinoma of the prostate. Cancer $1990 ; 66: 1225-33$.

2. Lee F, Littrup PJ, Loft-Christensen L, et al. Predicted prostate specific antigen results using transrectal ultrasound gland volume. Cancer $1992 ; 70$ (Suppl.) : 211-20.

3. Johansson JE, Admi HO, Andersson SO, Bergström R, Holmberg L, Krusemo UB. High 10-ycar survival rate in patients with early, untrcated prostatic cancer. JAMA 1992 ; 267 : 2191-6.

4. Larson A, Noren BJ. Fivc-ycar follow-up of patients with localized prostatic carcinoma initially referred for expectant treatment. Scand J Urol Nephrol 1985 ; 19 (Suppl. 93) : 30.

5. Epstcin JI, Paull G, Eggleston JC and Walsh PC. Prognosis of untreated stage $A_{1}$ prostatic carcinoma : a study of 94 cases with extended follow-up. J Urol $1986 ; 136$ : 837-9.

6. Labrie F, Dupont A, Suburu R, et al. Serum prostate specific antigen as prescreening test for prostate cancer. $J$ Uro $1992 ; 147: 846-52$

7. Jewett $\mathrm{HJ}$. Palpable nodule of prostatic cancer. JAMA 1968 ; 203: 403-6.

8. Walsh PC. Why make an early diagnosis of prostate cancer. J Urol $1992 ; 147$ : 853-4.

9. Labrie F. Endocrine therapy for prostate cancer. Endocrinol Metab Clin North Am 1991 ; 20 : 845-72.

\section{TIRÉS A PART}

F. Labrie. 